\title{
Dynamic Reconfiguration of the Supplementary Motor Area Network during Imagined Music Performance
}

\author{
Shoji Tanaka ${ }^{1 *}$ and Eiji Kirino ${ }^{2,3}$ \\ ${ }^{1}$ Department of Information and Communication Sciences, Sophia University, Tokyo, Japan, ${ }^{2}$ Department of Psychiatry, \\ School of Medicine, Juntendo University, Tokyo, Japan, ${ }^{3}$ Department of Psychiatry, Juntendo Shizuoka Hospital, Shizuoka, \\ Japan
}

The supplementary motor area (SMA) has been shown to be the center for motor planning and is active during music listening and performance. However, limited data exist on the role of the SMA in music. Music performance requires complex information processing in auditory, visual, spatial, emotional, and motor domains, and this information is integrated for the performance. We hypothesized that the SMA is engaged in multimodal integration of information, distributed across several regions of the brain to prepare for ongoing music performance. To test this hypothesis, functional networks involving the SMA were extracted from functional magnetic resonance imaging (fMRI) data that were acquired from musicians during imagined music performance and during the resting state. Compared with the resting condition, imagined music performance increased connectivity of the SMA with widespread regions in the brain including the sensorimotor cortices, parietal cortex, posterior temporal cortex, occipital cortex, and inferior and dorsolateral prefrontal cortex. Increased connectivity of the SMA with the

OPEN ACCESS

Edited by:

Nandini Chatterjee Singh,

National Brain Research Centre (NBRC), India

Reviewed by: Felipe Ortuño, Universidad de Navarra, Spain Masahiko Takada, Kyoto University, Japan

${ }^{*}$ Correspondence:

Shoji Tanaka shoji.tanaka@gmail.com

Received: 11 August 2017 Accepted: 28 November 2017 Published: 12 December 2017

Citation: Tanaka S and Kirino E (2017) Dynamic Reconfiguration of the Supplementary Motor Area Network during Imagined Music Performance.

Front. Hum. Neurosci. 11:606. doi: 10.3389/fnhum.2017.00606 dorsolateral prefrontal cortex suggests that the SMA is under cognitive control, while increased connectivity with the inferior prefrontal cortex suggests the involvement of syntax processing. Increased connectivity with the parietal cortex, posterior temporal cortex, and occipital cortex is likely for the integration of spatial, emotional, and visual information. Finally, increased connectivity with the sensorimotor cortices was potentially involved with the translation of thought planning into motor programs. Therefore, the reconfiguration of the SMA network observed in this study is considered to reflect the multimodal integration required for imagined and actual music performance. We propose that the SMA network construct "the internal representation of music performance" by integrating multimodal information required for the performance.

Keywords: emotion, functional connectivity, imagery, imagination, mental simulation, language, representation

\section{INTRODUCTION}

Music performance requires the integration of various kinds of information, including the sensory, motor, cognitive, and emotional domains related to the performance. This information has been shown to be distributed in the brain. In this article, we sought to analyze the dynamic reconfiguration of a functional network of the supplementary motor area (SMA) during imagined music performance to better understand the mechanism of multimodal integration in music performance. Because the SMA has been associated with motor planning, we hypothesized that 
the SMA network is engaged in multimodal integration, thereby mediating performance planning. The SMA lies in the caudal and medial part of the superior frontal gyrus, which is a medial part of Brodmann's area (BA) 6, anterior to the primary motor cortex or BA 4 (Nachev et al., 2008; Cona and Semenza, 2017). The SMA is activated by a range of tasks that require motor programming and execution. A functional magnetic resonance imaging (fMRI) study suggested that the SMA is substantially involved in the coordination of non-homologous limbs as part of a distributed motor network (Debaere et al., 2001). More recent studies suggest that the functions of SMA are not restricted to the domain of motor control and also include non-motor functions. For example, the SMA mediates sequence processing in various cognitive domains such as action sequences, time processing, spatial processing, numerical cognition, language, and music perception, and production (Hertrich et al., 2016; Cona and Semenza, 2017). The encoding and recall of visuospatial sequences are associated with SMA activation (Langner et al., 2014). The involvement of the SMA in working memory has been reported in auditory, visual, spatial, and object working memories (D’Esposito et al., 1998; Hautzel et al., 2002; Gruber and von Cramon, 2003), as well as arithmetic operations (Suchan et al., 2006; Fehr et al., 2007). These observations have led to the domain-general hypothesis, which states that the SMA plays a common role in all of these various domains. Specifically, the authors hypothesized that "the SMA is involved in domain-general sequence processes, contributing as a hub to the integration of elements into a sequence" (Cona and Semenza, 2017). Because sequence processing is essential in music performance, this notion is compatible with previous studies suggesting that the SMA is a core region for music processing (Bangert et al., 2006; Cona and Semenza, 2017).

Studies have shown that the SMA is involved in the production and perception of music (Janata and Grafton, 2003; Chen et al., 2008). Music performance requires action-oriented working memory (Langner et al., 2014) and goal-directed behavioral control (Spielberg et al., 2015). To this end, an fMRI study demonstrated the involvement of the SMA in working memory during a simple music listening task (Burunat et al., 2014). Another fMRI study implicated the SMA in the anticipation of sound sequences (Leaver et al., 2009). Furthermore, the "what, when, whether model" of intentional action suggests that the SMA plays an important role in the "when" component (Brass and Haggard, 2008). It has also been demonstrated that the SMA is involved in auditory-motor transformation (Chen et al., 2009), which is required for music performance. However, several questions regarding the role of the SMA in music processing and performance remain unanswered. This article employs functional connectivity analysis to obtain insight into the role of the SMA in music.

Functional connectivity in the brain can change rapidly during task performance (Braun et al., 2015; Fuertinger et al., 2015). This dynamic reconfiguration of functional networks has been studied using various tasks, including the n-back working memory task (Cohen et al., 2014; Braun et al., 2015; Cohen and D'Esposito, 2016) and speech control (Fuertinger et al., 2015; Simonyan and Fuertinger, 2015). These studies demonstrated the ability of the human brain to reconfigure its functional network dynamically, based on current cognitive demands (Cole et al., 2013; Cohen and D'Esposito, 2016). Based on this premise, the network configuration of the SMA may be subject to dynamic change during imagined music performance if the network is involved in the task. To date, functional connectivity studies of the SMA are limited. At rest, the SMA has been shown to have connections with the primary motor cortex (M1)/primary somatosensory cortex (S1), premotor cortex (PMC), middle frontal gyrus (MFG), orbitofrontal cortex (OFC), and thalamus (Zhang et al., 2012). In this study, we evaluated a functional network involving the SMA during imagined music performance and the resting state in order to observe dynamic reconfiguration of the SMA network. If the SMA increases its functional connectivity during imagined performance, the network would be considered to be utilized for processing information needed for the performance. We predicted that the active network during imagined performance would represent processes resembling actual music performance minus processes related to motor output control. Motor output was suppressed in our experiment since participants were lying in the MRI device and instructed to remain immobile. Because of this physical state, imaging will be able to dissociate non-motor from motor processes. We also predicted that the SMA network mediating cognitive processes not directly linked to motor control is enhanced during imagined performance compared with the resting state.

\section{MATERIALS AND METHODS}

\section{Ethical Issues}

All study procedures were approved by the ethics committees of Sophia University and Juntendo University and the study conformed to the tenets of the Declaration of Helsinki. All subjects provided written informed consent prior to study participation.

\section{Participants}

We recruited 41 graduate and undergraduate music school students (mean age, 23.4 years; age range, 19-30 years). All participants were healthy, right-handed, Japanese women, without a history of neurological or neuropsychiatric disease. Students majoring in music had begun musical training at the age of 3-5 years (i.e., all participants had more than 15 years of musical training) and had actively participated in concert performances. These students specialized in classical music played on various instruments: 15 played the piano, 8 the violin, 4 the clarinet, and 14 were opera vocalists.

\section{Tasks}

All participants completed two fMRI sessions: the first was the imagined music performance session followed by the restingstate session. Each session lasted $6 \mathrm{~min}$ and $40 \mathrm{~s}$. During the imagined music performance session, the participants were asked to imagine the act of music performance in a concert hall as vividly as possible without performing actual movements with their eyes closed. The music "performed" was chosen freely from their repertoires. For example, pianists chose a piece of piano 
music (e.g., Ballade No. 1 by Frederic Chopin), violinists chose a piece of violin music (e.g., Violin Sonata No. 1 by Robert Schumann), and vocalists chose an opera aria (e.g., "Regnava nel silenzio" from Lucia di Lammermoor by Donizetti). The performance was truncated at the end of each session. In the resting-state session, the participants were instructed not to think anything in particular with their eyes closed.

\section{Image Acquisition Structural Images}

Whole-brain images were acquired on a Philips Achieva 3.0T MRI scanner equipped with 32-channel head coils. Highresolution T1-weighted images were collected for anatomical reference using a 3D magnetization-prepared rapid acquisition gradient echo (MPRAGE) sequence: echo time $(\mathrm{TE})=3.3 \mathrm{~ms}$, repetition time $(\mathrm{TR})=15 \mathrm{~ms}$, flip angle $=10^{\circ}$, matrix size $=180$ $\times 256 \times 256$, and voxel size $=1 \times 1 \times 1 \mathrm{~mm}^{3}$. The total image acquisition time was $3 \mathrm{~min} 31 \mathrm{~s}$.

\section{Functional Images}

Blood-oxygen-level dependent (BOLD) fMRI data were collected during the imagined music performance session and the restingstate session. A T2*-weighted gradient-echo-planar imaging sequence was used with the following parameters: $\mathrm{TE}=30 \mathrm{~ms}$, $\mathrm{TR}=2,000 \mathrm{~ms}$, flip angle $=90^{\circ}$, field of view $=240 \times 240 \mathrm{~mm}^{2}$, matrix size $=64 \times 64$, number of axial slices $=33$, and voxel size $=3.75 \times 3.75 \times 4.00 \mathrm{~mm}^{3}$. Each session consisted of 200 scans. The image acquisition time was $6 \min 40 \mathrm{~s}$.

\section{Preprocessing}

Imaging data were preprocessed using the CONN toolbox (Whitfield-Gabrieli and Nieto-Castanon, 2012) run on MATLAB (R2016b, MathWorks, Inc.). The individual fMRI data were coregistered to the $\mathrm{T} 1$ images. The first four volumes were discarded and the remaining 196 volumes were subjected to preprocessing. The fMRI data were slice-timed, realigned, and subsequently normalized to the standard Montreal Neurological Institute (MNI) template as implemented in the Statistical Parametric Mapping (SPM) software platform. Image artifacts originating from head movement were processed using the ART scrubbing procedure. Signal contributions from white matter, cerebrospinal fluid, and micro head movements (six parameters) were regressed out from the data. Finally, the fMRI data were band-pass filtered $(0.008-0.09 \mathrm{~Hz})$ and functional images were spatially smoothed using a Gaussian filter kernel (full width at half-maximum = $8 \mathrm{~mm}$ ) for the subsequent seed-to-voxel analysis.

\section{Statistical Analyses}

A seed-to-voxel functional connectivity analysis was performed using the CONN toolbox. The seed was either the left or right SMA. Pearson's correlation coefficients were calculated between the time course of the left or right SMA and the time courses of all other voxels in the gray matter, which provided a seedto-voxel connectivity matrix. Positive and negative correlation coefficients defined positive and negative functional connectivity, respectively (Whitfield-Gabrieli and Nieto-Castanon, 2012). The correlation coefficients were then converted to normally distributed scores using Fisher's transformation and subsequently used in the population-level analysis. A height threshold of $p$ $<0.001$, uncorrected, was applied to individual voxels to define clusters. The extracted clusters were then set to $p<0.05$ with the family-wise error (FWE) correction for results reporting.

\section{RESULTS}

To explore how the SMA network is reconfigured during imagined music performance, we analyzed functional connectivity of the SMA during both imagined music performance and the resting sate. The analysis extracted significantly enhanced functional network of the SMA. Figures 1, 2 show the results of the seed-to-voxel functional connectivity analysis. Lists of the clusters of voxels whose functional connectivity with the SMA differed significantly between the imagined music performance condition and the resting condition are given in Tables 1, 2. The results showed that the bilateral SMA exhibited enhanced functional connectivity with clusters of voxels in the superior parietal lobule (SPL), superior lateral occipital cortex (sLOC), precuneus, precentral gyrus (preCG), postcentral gyrus (postCG), supramarginal gyrus (SMG), inferior frontal gyrus (IFG), posterior temporal and occipital regions, and cerebellum during imagined music performance compared with the resting state $(p<0.05$, FWE). The posterior temporal regions include the posterior superior temporal gyrus (pSTG), posterior middle temporal gyrus (pMTG), temporo-occipital middle temporal gyrus (toMTG), and temporo-occipital inferior temporal gyrus (toITG). Connectivity of the left SMA with the clusters of voxels in the bilateral superior frontal gyrus (SFG), MFG, and caudate nucleus and the right SMA with the clusters of voxels in the bilateral frontal pole (FP) was also increased during imagined music performance. On the other hand, the left SMA showed decreased connectivity with the voxels in the right central operculum during imagined performance compared with the resting condition.

\section{DISCUSSION}

\section{Motor Coordination}

\section{The Superior Parietal Lobule}

We identified increased connectivity of the SMA with the SPL and its posterior extension into the occipital cortex, the sLOC, during imagined music performance. The SPL receives input from the visual cortex and somatosensory cortex. Accordingly, the SPL processes visuospatial imagery, attention, and working memory (Schacter et al., 2012; Spreng et al., 2013). The anterior part of BA 7, a part of the SPL, has been implicated in the sequencing of hand actions (Heim et al., 2012). This area has also been previously associated with speech production, and is therefore considered to be involved in general motor sequencing. The left SPL has been associated with tool use (Garcea and Mahon, 2014). Typewriting and handwriting activate the SPL, SMG, and left premotor cortex close to Exner's area (Planton et al., 2013; Higashiyama et al., 2015). A structural equation modeling study showed that the SPL contributes via inputs to the SMA to the formation of motor imagery during a task conceiving 

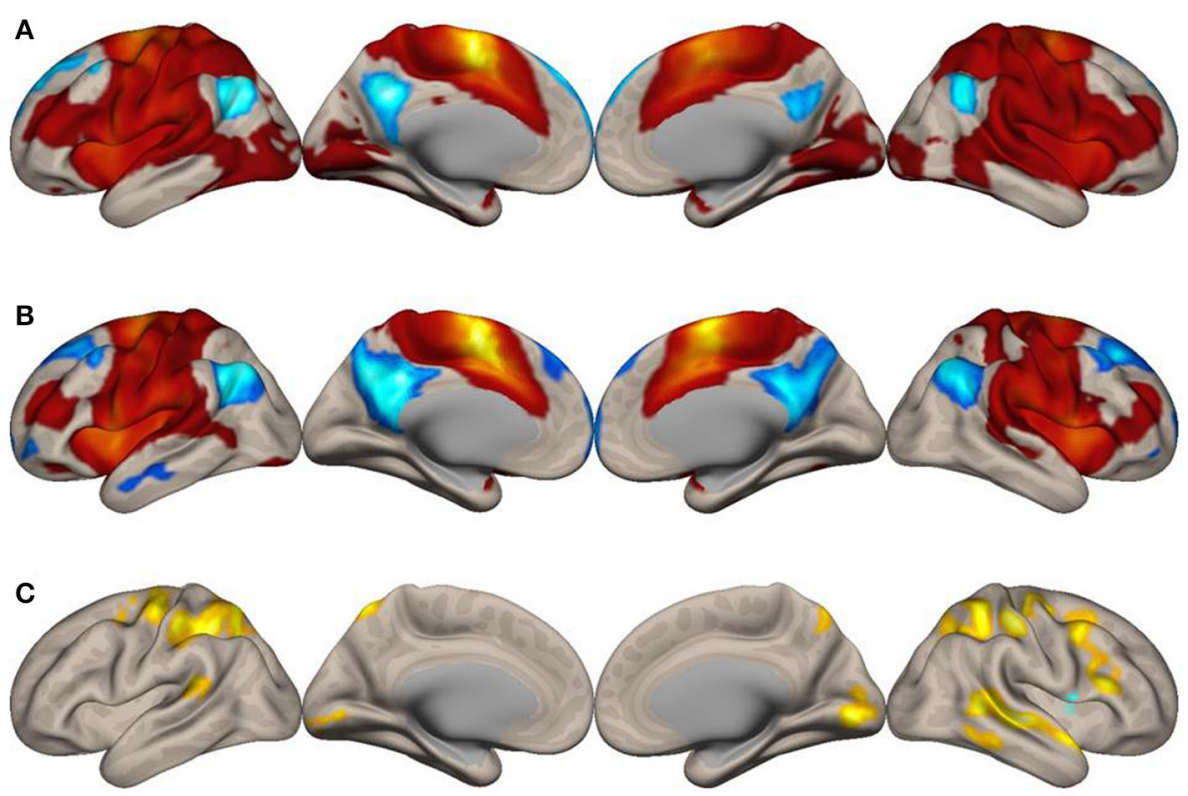

FIGURE 1 | Surface maps of functional connectivity of the left supplementary motor area (SMA) as the seed region. (A) Imagined music performance. (B) Resting state. Voxels with positive connectivity are colored in red and voxels with negative connectivity are colored in blue. (C) Voxels with significant differences in connectivity with the left SMA between the imagined music performance and resting-state conditions are in yellow to indicate higher connectivity and blue lower connectivity during the performance condition, compared with the resting condition. The significance level was peak-voxel $p<0.001$ ( $T \geq 3.55$ ), uncorrected, and cluster $p<$ 0.05 , family-wise error corrected.
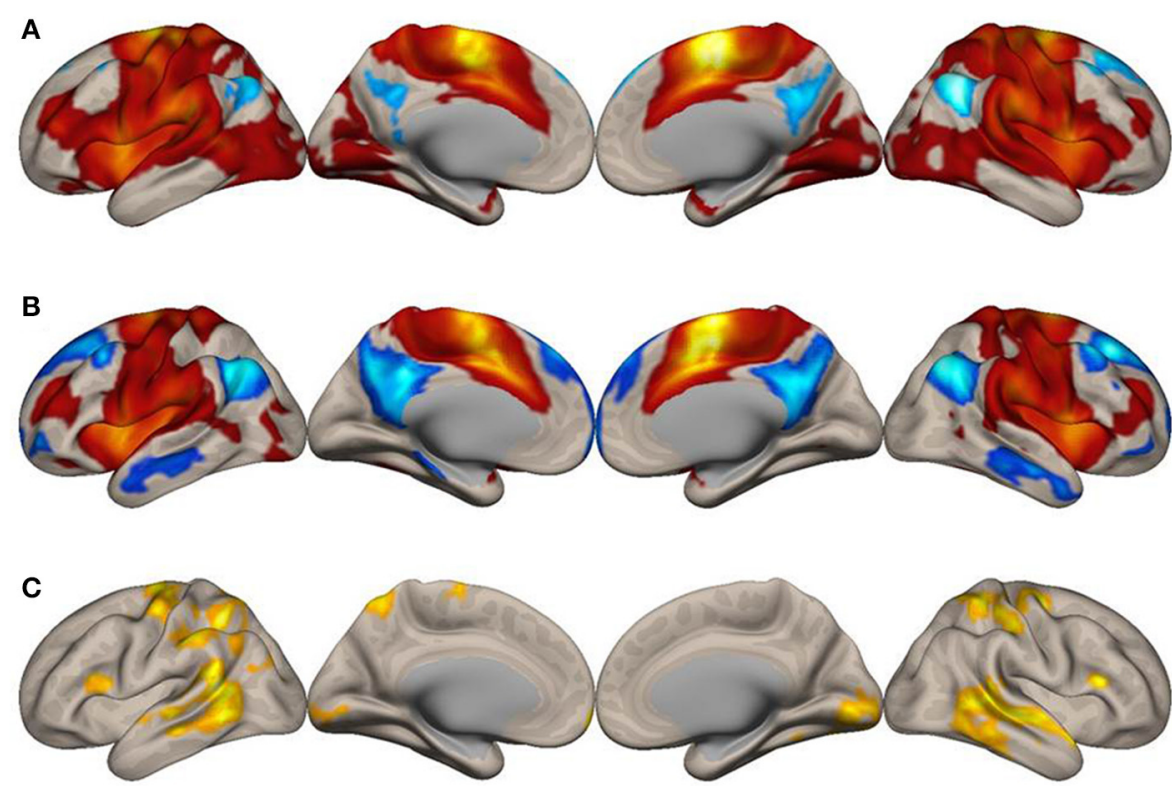

FIGURE 2 | Surface maps of functional connectivity of the right supplementary motor area (SMA) as the seed region. All conventions are as in Figure 1.

visual or kinesthetic imagery of unpaced thumb-opposition movement (Solodkin et al., 2004). In general, the posterior parietal cortex has been associated with the planning and control of goal-directed action (Vesia and Crawford, 2012). The finding that the SPL mediates motor sequencing in the hand and speech domains (Heim et al., 2012) has important implications for its association with music processing. The SPL is consistently activated during covert musical rehearsal (Langheim et al., 2002), 
TABLE 1 | Clusters of voxels whose functional connectivity with the left supplementary motor area (SMA) differed significantly between the imagined music performance condition and the resting condition.

\begin{tabular}{llcl}
\hline Cluster $(\mathbf{x}, \mathbf{y}, \mathbf{z})$ & Size & Cluster $\boldsymbol{p}$-FWE & Peak $\boldsymbol{p}$-un \\
\hline$(-48-34+48)$ & 9147 & 0.0000 & 0.000000 \\
$(+56-24-04)$ & 1648 & 0.0000 & 0.000000 \\
$(+04-84+00)$ & 705 & 0.0000 & 0.000001 \\
$(-16-70-30)$ & 581 & 0.0001 & 0.000001 \\
$(+18-46-22)$ & 511 & 0.0003 & 0.000001 \\
$(+10+06+00)$ & 501 & 0.0004 & 0.000019 \\
$(-52-40+16)$ & 326 & 0.0052 & 0.000011 \\
$(-18+10+12)$ & 285 & 0.0103 & 0.000019 \\
$(+58-40-14)$ & 222 & 0.0312 & 0.000079 \\
$(+34+04+12)$ & 197 & 0.0496 & 0.000998
\end{tabular}

Cluster 1

- 1001 voxels covering 20\% of atlas.sLOC I (Lateral Occipital Cortex, superoir division Left)

- 854 voxels covering 20\% of atlas.PreCG I (Precentral Gyrus Left)

- 838 voxels covering 57\% of atlas.SPL I (Superior Parietal Lobule Left)

- 790 voxels covering 54\% of atlas.SPL r (Superior Parietal Lobule Right)

- 726 voxels covering 26\% of atlas.MFG r (Middle Frontal Gyrus Right)

- 719 voxels covering 15\% of atlas.sLOC r (Lateral Occipital Cortex, superoir division Right)

- 505 voxels covering 12\% of atlas.PreCG r (Precentral Gyrus Right)

- 491 voxels covering 13\% of atlas.PostCG I (Postcentral Gyrus Left)

- 438 voxels covering 14\% of atlas.PostCG r (Postcentral Gyrus Right)

- 396 voxels covering $42 \%$ of atlas.aSMG I (Supramarginal Gyrus, anterior division Left)

- 214 voxels covering 4\% of atlas.Precuneus (Precuneus Cortex)

- 211 voxels covering 20\% of atlas.pSMG I (Supramarginal Gyrus, posterior division Left)

- 186 voxels covering 15\% of atlas.pSMG r (Supramarginal Gyrus, posterior division Right)

- 177 voxels covering 6\% of atlas.MFG I (Middle Frontal Gyrus Left)

- 166 voxels covering 24\% of atlas.IFG oper r (Inferior Frontal Gyrus, pars opercularis Right)

- 139 voxels covering 5\% of atlas.SFG r (Superior Frontal Gyrus Right)

- 131 voxels covering 24\% of atlas.IFG tri r (Inferior Frontal Gyrus, pars triangularis Right)

- 105 voxels covering 13\% of atlas.aSMG r (Supramarginal Gyrus, anterior division Right)

- 67 voxels covering 2\% of atlas.SFG I (Superior Frontal Gyrus Left)

Cluster 2

- 394 voxels covering 34\% of atlas.toMTG r (Middle Temporal Gyrus, temporooccipital division Right)

- 306 voxels covering 23\% of atlas.pMTG r (Middle Temporal Gyrus, posterior division Right)

- 182 voxels covering 44\% of atlas.pSTG r (Superior Temporal Gyrus, posterior division Right)

- 115 voxels covering 41\% of atlas.aSTG r (Superior Temporal Gyrus, anterior division Right)

- 98 voxels covering 4\% of atlas.TP r (Temporal Pole Right)

- 98 voxels covering 8\% of atlas.pSMG r (Supramarginal Gyrus, posterior division Right)
TABLE 1 | Continued

\begin{tabular}{|c|c|c|c|}
\hline Cluster $(x, y, z)$ & Size & Cluster $p$-FWE & Peak $p$-unc \\
\hline $\begin{array}{l}\text { Cluster } 3 \\
\text { - } 193 \text { voxels } \\
\text { - } 176 \text { voxels } \\
\text { - } 73 \text { voxels cc } \\
\text { - } 59 \text { voxels cc }\end{array}$ & $\begin{array}{l}\% \text { of atl } \\
\% \text { of atl } \\
\text { f atlas. } \\
\text { f atlas. }\end{array}$ & $\begin{array}{l}\text { Lingual Gyrus Righ } \\
\text { (Intracalcarine Cor } \\
\text { cipital Pole Right) } \\
\text { cipital Pole Left) }\end{array}$ & ght) \\
\hline $\begin{array}{l}\text { Cluster } 4 \\
\text { - } 157 \text { voxels } \\
\text { - } 62 \text { voxels cc } \\
\text { - } 54 \text { voxels cc }\end{array}$ & $\begin{array}{l}\text { of atla } \\
\text { f atlas. } \\
\text { f atlas. }\end{array}$ & $\begin{array}{l}\text { I (Cerebellum Crus } \\
\text { (Cerebellum } 6 \text { Lef } \\
\text { I (Cerebellum } 45\end{array}$ & \\
\hline $\begin{array}{l}\text { Cluster } 5 \\
\text { - } 176 \text { voxels } \\
\text { - } 126 \text { voxels }\end{array}$ & $\begin{array}{l}\% \text { of atl } \\
\text { of atlas }\end{array}$ & $\begin{array}{l}45 \text { r (Cerebellum } \\
\text { r (Cerebellum } 6 \text { R }\end{array}$ & ght) \\
\hline \multicolumn{4}{|c|}{ - 223 voxels covering 43\% of atlas.Caudate r (Caudate Nucleus Right) } \\
\hline \multicolumn{3}{|c|}{$\begin{array}{l}\text { - } 88 \text { voxels covering } 8 \% \text { of atlas.pSMG I (Supramarginal Gyrus, posterior } \\
\text { division Left) }\end{array}$} & osterior \\
\hline \multicolumn{3}{|c|}{ Cluster 8} & eft) \\
\hline \multicolumn{4}{|c|}{$\begin{array}{l}\text { - } 150 \text { voxels covering } 19 \% \text { of atlas.tolTG r (Inferior Temporal Gyrus, } \\
\text { temporooccipital part Right) }\end{array}$} \\
\hline \multicolumn{4}{|l|}{ Cluster 10} \\
\hline
\end{tabular}

Only the regions whose cluster size were larger than or equal to 50 were listed.

which is similar to the task employed in this study. The SPL is also activated during music sight-reading (Schon et al., 2002) and mental reversal of imagined melodies (Zatorre et al., 2010). Therefore, consistent with the findings of previous studies, our finding of increased functional connectivity between the SMA and SPL/sLOC during imagined music performance suggests that the SMA network mediates information processing related to intended music performance, including intended manipulation of an instrument.

\section{The Pre/Postcentral Gyrus and Cerebellum}

Connectivity of the SMA with the pre/postCG and cerebellum was significantly increased during imagined music performance. The preCG or motor cortex is traditionally thought to control voluntary ongoing movements (Tomasino and Gremese, 2016). Thus, our result was unexpected given that participants were not allowed to execute movements during the task. The pre/postCG or sensorimotor areas are principal regions for voluntary movement control (Tomasino and Gremese, 2016). Therefore, this result can be interpreted as follows: first, connections between the SMA and motor regions could represent a pathway involved in the translation of an intended action into a motor program. Second, it is possible that the SMA and motor cortex worked cooperatively to dissociate body movements from the imagined performance. During the imagined performance task, participants had to make conscious efforts not to move their 
TABLE 2 | Clusters of voxels whose functional connectivity with the right supplementary motor area (SMA) differed significantly between the imagined music performance condition and the resting condition.

\begin{tabular}{llcl}
\hline Cluster $(\mathbf{x}, \mathbf{y}, \mathbf{z})$ & Size & Cluster $\boldsymbol{p}$-FWE & Peak $\boldsymbol{p}$-un \\
\hline$(-56-50+12)$ & 6977 & 0.0000 & 0.000000 \\
$(+52-30-04)$ & 3030 & 0.0000 & 0.000001 \\
$(+26-10+60)$ & 1971 & 0.0000 & 0.000000 \\
$(+16-50-30)$ & 1835 & 0.0000 & 0.000001 \\
$(-02+68-12)$ & 443 & 0.0009 & 0.000001 \\
$(+56+26+20)$ & 256 & 0.0175 & 0.000001 \\
$(-50+18+16)$ & 228 & 0.0288 & 0.000048
\end{tabular}

Cluster 1

- 1193 voxels covering 24\% of atlas.sLOC I (Lateral Occipital Cortex, superoir division Left)

- 821 voxels covering 19\% of atlas.PreCG I (Precentral Gyrus Left)

- 727 voxels covering 84\% of atlas.toMTG I (Middle Temporal Gyrus, temporooccipital part Left)

- 565 voxels covering 53\% of atlas.pSMG I (Supramarginal Gyrus, posterior division Left)

- 551 voxels covering 38\% of atlas.SPL I (Superior Parietal Lobule Left)

- 543 voxels covering $57 \%$ of atlas.aSMG I (Supramarginal Gyrus, anterior division Left)

- 352 voxels covering 25\% of atlas.pMTG I (Middle Temporal Gyrus, posterior division Left)

- 285 voxels covering 30\% of atlas.AG I (Angular Gyrus Left)

- 246 voxels covering 7\% of atlas.PostCG I (Postcentral Gyrus Left)

- 226 voxels covering 4\% of atlas.Precuneus (Precuneus Cortex)

- 132 voxels covering 23\% of atlas.PT I (Planum Temporale Left)

- 122 voxels covering 31\% of atlas.pSTG I (Superior Temporal Gyrus, posterior division Left)

- 111 voxels covering 5\% of atlas.iLOC I (Lateral Occipital Cortex, inferior division Left)

Cluster 2

- 734 voxels covering 63\% of atlas.toMTG r (Middle Temporal Gyrus, temporooccipital part Right)

- 447 voxels covering 33\% of atlas.pMTG r (Middle Temporal Gyrus, posterior division Right)

- 367 voxels covering 47\% of atlas.tolTG r (Inferior Temporal Gyrus, temporooccipital part Right)

- 210 voxels covering 50\% of atlas.pSTG r (Superior Temporal Gyrus, posterior division Right)

- 132 voxels covering 47\% of atlas.aSTG r (Superior Temporal Gyrus, anterior division Right)

- 116 voxels covering 12\% of atlas.pITG r (Inferior Temporal Gyrus, posterior division Right)

- 114 voxels covering 9\% of atlas.pSMG r (Supramarginal Gyrus, posterior division Right)

- 112 voxels covering 5\% of atlas.TP r (Temporal Pole Right)

- 50 voxels covering $12 \%$ of atlas.aMTG r (Middle Temporal Gyrus, anterior division Right)

Cluster 3

- 644 voxels covering 20\% of atlas.PostCG r (Postcentral Gyrus Right)

- 576 voxels covering 39\% of atlas.SPL r (Superior Parietal Lobule Right)

- 506 voxels covering 12\% of atlas.PreCG r (Precentral Gyrus Right)

- 81 voxels covering $10 \%$ of atlas.aSMG r (Supramarginal Gyrus, anterior division Right)
TABLE 2 | Continued

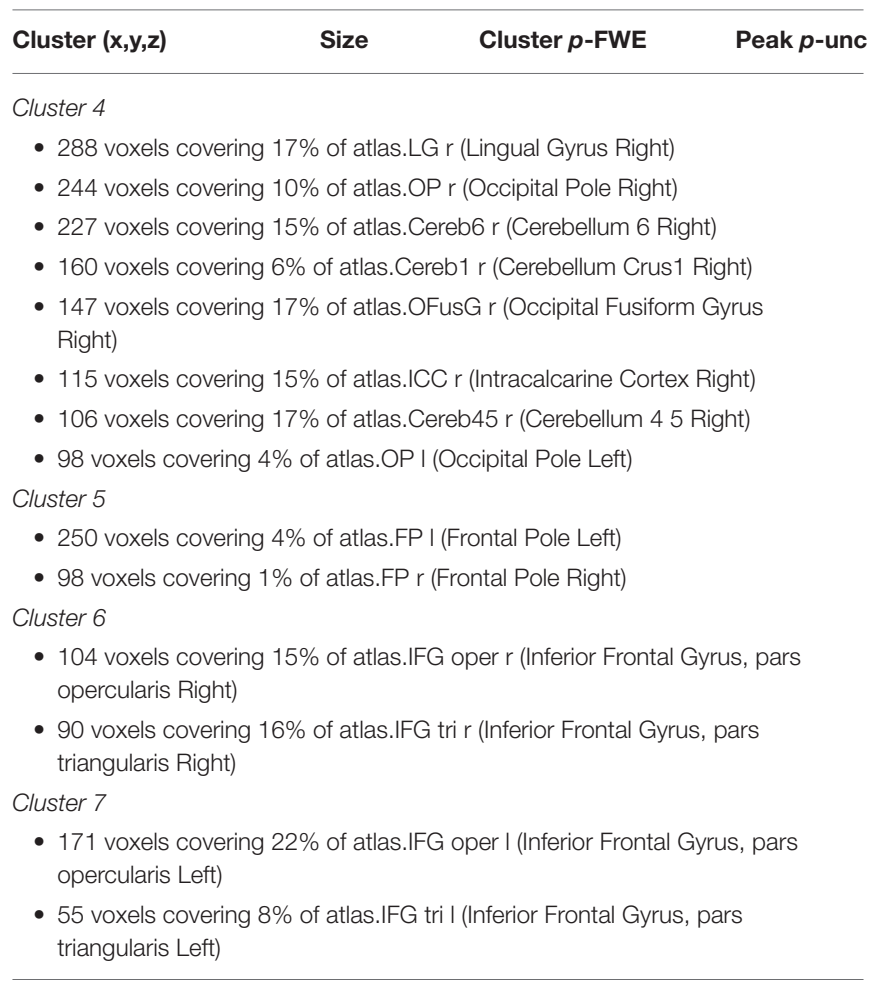

Only the regions whose cluster size were larger than or equal to 50 were listed.

body parts; thus, increased connectivity between the SMA and motor regions may have been utilized to inhibit involuntary body movements. Third, given that the motor cortex is involved in cognitive as well as motor processing (Leisman et al., 2016), connectivity between the SMA and motor cortex may represent an avenue of motor-cognitive processing. A recent metaanalysis identified activation of the motor cortex in response to six cognitive functional categories: motor imagery, working memory, mental rotation, social/emotion/empathy, language, and auditory processing (Tomasino and Gremese, 2016). If the motor cortex mediates cognitive processing (Tomasino and Gremese, 2016), the motor cortex would accomplish this by functional networking with the SMA and other related areas.

\section{The Perisylvian Network}

In the present study, the SMA exhibited increased connectivity with the SMG during imagined music performance. The increased connectivity with the left SMG was more marked than that with the right SMG for both the left and right SMA. The left SMG is a node of the dorsal language pathway (Catani et al., 2005; Gierhan, 2013) and involved in speech production and perception as well as verbal working memory (Hartwigsen et al., 2010; Turkeltaub and Coslett, 2010; Zevin et al., 2010; Deschamps et al., 2014; Simmonds et al., 2014; Simonyan and Fuertinger, 2015). One fMRI study demonstrated that the SMG was activated during immersive second language learning in monolingual adults, and that activity was positively correlated with second-language acquisition capability (Barbeau et al., 2016). In a study of bilingual individuals, functional 
connectivity between the SMG and IFG was enhanced in those who became simultaneously bilingual, compared with those who became sequentially bilingual, and the degree of connectivity was correlated with the age of second language acquisition in sequential bilingual individuals (Berken et al., 2016). Music performance also requires cooperating auditory and motor processing. This is evidenced by fMRI studies indicating the involvement of the SMG in music processing using auditory and motor tasks in professional pianists (Bangert et al., 2006; Bengtsson and Ullén, 2006). The SMG is also associated with pitch memory and regulation (Zarate and Zatorre, 2008; Schaal et al., 2015a,b). Therefore, our finding of increased functional connectivity between the SMA and SMG during imagined performance is consistent with previous findings, supporting a relationship between music performance and speech production as suggested in previous studies (Koelsch et al., 2002; Brown et al., 2006). The SMG participates with the IFG in a network that mediates the abstract coding of audiovisual speech (Hasson et al., 2007). Studies suggest that the IFG, especially in the right hemisphere, mediates music syntax processing (Koelsch, 2005), and that the volume of the gray matter in the right IFG is larger in musicians than in non-musicians (Sato et al., 2015). The IFG is structurally and functionally connected with the SMG and STG (Kelly et al., 2010), forming a part of the perisylvian network for language processing (Catani et al., 2005; Xiang et al., 2010; Friederici, 2011; Gierhan, 2013). It should be noted that the connectivity of the SMA with the IFG, SMG, and STG was increased during imagined music performance. To our knowledge, our functional connectivity analysis is the first to show the recruitment of this network by the SMA during imagined music performance. Thus, it appears that the SMA contributes to music syntax processing during imagined music performance by interacting with the perisylvian network. In contrast to language syntax processing (den Ouden et al., 2012), the increased connectivity between the SMA and perisylvian network was not restricted to the left hemisphere. It seems that the network for music syntax processing is bilateral or even rightward lateralized, as indicated by rightward lateralized activation during music syntax processing (Koelsch, 2005; Musso et al., 2015). It should be noted, however, that there remains a debate concerning the laterality of syntax processing in music (Patel, 2003; Asano and Boeckx, 2015). A recent study suggests a common syntax processing role of the left IFG or the Broca's area in music and language (Kunert et al., 2015). Further investigations are needed for elucidating the network for music syntax processing.

\section{Visual Imagery}

Our analysis showed increased connectivity of the SMA with the precuneus, PCC, lateral occipital cortex (LOC), lingual gyrus, intracalcarine cortex, and OP during imagined music performance. The precuneus and PCC have been previously associated with the mental representation of visual scenes (Cavanna and Trimble, 2006; Johnson and Johnson, 2014; Tanaka and Kirino, 2016, 2017). Therefore, connecting with visual areas may enable the SMA to process mental imagery of body movements and visual scenes. The LOC includes the middle temporal (MT)/V5 and extrastriate body area (EBA). The MT/V5 mediates visual motion perception (Born and Bradley, 2005), generally showing higher responses to moving body parts than to moving objects (Spiridon et al., 2006). The EBA mediates the visual perception of body parts (Astafiev et al., 2004) and is thought to contribute to understanding goal-directed actions by representing dynamic aspects of human motion (Takahashi et al., 2008). Activity of the EBA is modulated by limb (arm and foot) movements and by motor imagery without actual movements (Astafiev et al., 2004). Based on this premise, increased connectivity between the right SMA and visual cortex is intriguing, as participants kept their eyes closed during the imaging session. Increased connectivity between the SMA and these visual areas suggests that imagined music performance includes the processing of visual scene and imagined body movements.

\section{Semantic and Socio-Emotional Processing}

The SMA showed increased functional connectivity with posterior temporal regions (pSTS, pMTG, toMTG) during imagined music performance. Activation of the pSTS/MTG has been associated with audiovisual semantic integration ( $\mathrm{Li}$ et al., 2016). The pMTG is thought to be relatively specialized for semantic control (Noonan et al., 2013). Indeed, posterior regions of temporal lobe (pMTG and toMTG) contribute to semantic cognition and control (Whitney et al., 2011a,b; Jefferies, 2013; Davey et al., 2015, 2016). Semantic control and action understanding are processed by a network that includes the pMTG (Davey et al., 2015, 2016). The pMTG represents object properties (Martin, 2007), and semantic judgments about manipulable objects are represented in a network that includes the pMTG (Davey et al., 2015). The pMTG is functionally connected to regions of the frontoparietal network and shows increased activity during semantically demanding tasks (Tune and Asaridou, 2016). Accordingly, the pMTG has been associated with tool and action knowledge (Martin, 2007; van Elk et al., 2014). An fMRI study demonstrated that the pSTS was activated during the multimodal perception of nonverbal vocal and facial emotional signals and associated trait emotional intelligence with activity in the right pSTS (Kreifelts et al., 2010). In an experiment using naturalistic audiovisual movie clips, the pSTS was suggested to be a hub for social perception (Lahnakoski et al., 2012). Another study reported right hemisphere dominance for the emotional processing of scenes with social contents (Calvo et al., 2015). Consistent with this finding, the increased connectivity between the SMA and the posterior temporal regions in the present study has revealed to be rightward lateralized. Taken together, enhanced connectivity between the SMA and posterior temporal regions suggests that semantic and socio-emotional processing is included in the SMA network for imagined music performance.

\section{Dynamic Mental Representation of Music Performance}

A major question addressed in this study was the exact role of the SMA network during imagined music performance. Our result that the SMA increased its functional connectivity with many brain regions during imagined performance is consistent with the idea that the SMA integrates multimodal information required 
for the performance. The left SMA showed increased functional connectivity with lateral prefrontal regions (SFG/MFG/IFG), especially in the posterior regions, during our study task. These regions have been associated with central executive functions and working memory manipulation as well as maintenance (Nee and D'Esposito, 2016). Moreover, the right lateral prefrontal regions contribute to episodic memory retrieval: The activation of the right prefrontal cortex (PFC) is a consistent finding in neuroimaging studies of retrieval from episodic memory (Henson et al., 1999; Allan et al., 2000; Nyberg et al., 2000). Therefore, the increased connectivity between the SMA and the lateral prefrontal regions, especially in the right hemisphere, suggests that, under top-down control by the central executive system, the SMA construct "the internal representation of music performance" for the upcoming performance by integrating multimodal information represented in several brain regions. Just as working memory is not localized to a single brain region (D'Esposito, 2007), the executive imagery for music performance may be an emergent property of the interaction between the PFC and the SMA. This view is consistent with the rostro-caudal gradient organization theory of PFC functions, in which the posterior PFC represents behavioral and motor information (Badre, 2008; Depue et al., 2016). Complex cognitive control and time estimation, which are also required in music performance, engage the overlapping brain regions including the SMA, PMC, SPL, and precuneus (Radua et al., 2014). Our result is consistent with their meta-analysis. The enhanced network of the SMA during imagined music performance also include the temporal and occipital regions. The inclusion of these regions into the network indicates that the network mediates multimodal integration of the information needed for performance, specifically, the integration of visual imagery, syntactic, semantic, and socio-emotional information into motor imagery. Because various motor imagery tasks activate brain regions including the SMA, SPL, IFG, and preCG (Hanakawa et al., 2008; Hétu et al., 2013), our results suggest that the SMA network processes motor imagery. Therefore, we believe that information processing by the enhanced SMA network mediates multimodal integration into motor imagery for imagined music performance. Regarding functional networks, however, no comparison has ever been made between imagined and actual music performance. To date, an fMRI study reported

\section{REFERENCES}

Allan, K., Dolan, R. J., Fletcher, P. C., and Rugg, M. D. (2000). The role of the right anterior prefrontal cortex in episodic retrieval. Neuroimage 11, 217-227. doi: 10.1006/nimg.2000.0531

Asano, R., and Boeckx, C. (2015). Syntax in language and music: what is the right level of comparison? Front. Psychol. 6:942. doi: 10.3389/fpsyg.2015. 00942

Astafiev, S. V., Stanley, C. M., Shulman, G. L., and Corbetta, M. (2004). Extrastriate body area in human occipital cortex responds to the performance of motor actions. Nat. Neurosci. 7, 542-548. doi: 10.1038/nn1241

Badre, D. (2008). Cognitive control, hierarchy, and the rostro caudal organization of the frontal lobes. Trends Cogn. Sci. 12, 193-200. doi: 10.1016/j.tics.2008.02.004 that imagined and actual playing of short piano pieces in pianists activated brain regions in common: the sensorimotor, premotor, posterior parietal, and visual cortical areas, precuneus, and cerebellum (Meister et al., 2004). Among these regions, the sensorimotor and posterior parietal cortical areas were activated more during actual playing, which suggests that motor execution required the higher level of visuomotor integration.

\section{CONCLUSION}

We have characterized dynamic network reconfiguration of the SMA for imagined music performance. Functional connectivity between the SMA and regions distributed over the parietal, temporal, occipital, and frontal cortices as well as the cerebellum is increased during imagined music performance, compared with the resting state. The SMA processes multimodal information including visuospatial, motor, syntax, semantic, and socioemotional information relevant to intended music performance. We propose that the SMA network dynamically construct "the internal representation of music performance" by integrating multimodal information into motor imagery required for the performance.

\section{AUTHOR CONTRIBUTIONS}

ST and EK planned and conducted all the experiments. ST analyzed the data and wrote the manuscript.

\section{FUNDING}

This work was supported by JSPS KAKENHI Grant Number $15 \mathrm{~K} 00380$.

\section{ACKNOWLEDGMENTS}

The authors wish to thank all the students who participated in this study and the Toho Gakuen School of Music for their continuous support during the execution of this study. The authors thank Mayuko Fukuta, Rie Inami, and Masatoshi Hirayama at the Juntendo University School of Medicine for their technical assistance in the experiments.
Bangert, M., Peschel, T., Schlaug, G., Rotte, M., Drescher, D., Hinrichs, H., et al. (2006). Shared networks for auditory and motor processing in professional pianists: evidence from fMRI conjunction. Neuroimage 30, 917-926. doi: 10.1016/j.neuroimage.2005.10.044

Barbeau, E. B., Chai, X. J., Chen, J.-K., Soles, J., Berken, J., Baum, S., et al. (2016). The role of the left inferior parietal lobule in second language learning: An intensive language training fMRI study. Neuropsychologia 98, 169-176. doi: 10.1016/j.neuropsychologia.2016.10.003

Bengtsson, S. L., and Ullén, F. (2006). Dissociation between melodic and rhythmic processing during piano performance from musical scores. Neuroimage 30, 272-284. doi: 10.1016/j.neuroimage.2005.09.019

Berken, J. A., Chai, X., Chen, J.-K., Gracco, V. L., and Klein, D. (2016). Effects of early and late bilingualism on resting-state functional connectivity. J. Neurosci. 36, 1165-1172. doi: 10.1523/JNEUROSCI.1960-15.2016 
Born, R. T., and Bradley, D. C. (2005). Structure and function of visual area MT. Annu. Rev. Neurosci. 28, 157-189. doi: 10.1146/annurev.neuro.26.041002.131052

Brass, M., and Haggard, P. (2008). The what, when, whether model of intentional action. Neuroscientist 14, 319-325. doi: 10.1177/1073858408317417

Braun, U., Schäfer, A., Walter, H., Erk, S., Romanczuk-Seiferth, N., Haddad, L., et al. (2015). Dynamic reconfiguration of frontal brain networks during executive cognition in humans. Proc. Natl. Acad. Sci. U.S.A. 112, 11678-11683. doi: $10.1073 /$ pnas. 1422487112

Brown, S., Martinez, M. J., and Parsons, L. M. (2006). Music and language side by side in the brain: a PET study of the generation of melodies and sentences. Eur. J. Neurosci. 23, 2791-2803. doi: 10.1111/j.1460-9568.2006.04785.x

Burunat, I., Alluri, V., Toiviainen, P., Numminen, J., and Brattico, E. (2014). Dynamics of brain activity underlying working memory for music in a naturalistic condition. Cortex 57, 254-269. doi: 10.1016/j.cortex.2014.04.012

Calvo, M. G., Rodríguez-Chinea, S., and Fernández-Martín, A. (2015). Lateralized discrimination of emotional scenes in peripheral vision. Exp. Brain Res. 233, 997-1006. doi: 10.1007/s00221-014-4174-8

Catani, M., Jones, D. K., and ffytche, D. H. (2005). Perisylvian language networks of the human brain. Ann. Neurol. 57, 8-16. doi: 10.1002/ana.20319

Cavanna, A. E., and Trimble, M. R. (2006). The precuneus: a review of its functional anatomy and behavioural correlates. Brain 129, 564-583. doi: 10.1093/brain/awl004

Chen, J. L., Penhune, V. B., and Zatorre, R. J. (2008). Listening to musical rhythms recruits motor regions of the brain. Cereb. Cortex 18, 2844-2854. doi: 10.1093/cercor/bhn042

Chen, J. L., Penhune, V. B., and Zatorre, R. J. (2009). The Role of auditory and premotor cortex in sensorimotor transformations. Ann. N. Y. Acad. Sci. 1169, 15-34. doi: 10.1111/j.1749-6632.2009.04556.x

Cohen, J. R., and D'Esposito, M. (2016). The Segregation and integration of distinct brain networks and their relationship to cognition. J. Neurosci. 36, 12083-12094. doi: 10.1523/JNEUROSCI.2965-15.2016

Cohen, J. R., Gallen, C. L., Jacobs, E. G., Lee, T. G., and D’Esposito, M. (2014). Quantifying the reconfiguration of intrinsic networks during working memory. PLoS ONE 9:e106636. doi: 10.1371/journal.pone.0106636

Cole, M. W., Reynolds, J. R., Power, J. D., Repovs, G., Anticevic, A., and Braver, T. S. (2013). Multi-task connectivity reveals flexible hubs for adaptive task control. Nat. Neurosci. 16, 1348-1355. doi: 10.1038/nn.3470

Cona, G., and Semenza, C. (2017). Supplementary motor area as key structure for domain-general sequence processing: a unified account. Neurosci. Biobehav. Rev.72, 28-42. doi: 10.1016/j.neubiorev.2016.10.033

Davey, J., Rueschemeyer, S.-A., Costigan, A., Murphy, N., Krieger-Redwood, K., Hallam, G., et al. (2015). Shared neural processes support semantic control and action understanding. Brain Lang. 142, 24-35. doi: 10.1016/j.bandl.2015.01.002

Davey, J., Thompson, H. E., Hallam, G., Karapanagiotidis, T., Murphy, C., De Caso, I., et al. (2016). Exploring the role of the posterior middle temporal gyrus in semantic cognition: integration of anterior temporal lobe with executive processes. Neuroimage 137, 165-177. doi: 10.1016/j.neuroimage.2016.05.051

Debaere, F., Swinnen, S. P., Béatse, E., Sunaert, S., Van Hecke, P., and Duysens, J. (2001). Brain areas involved in interlimb coordination: a distributed network. Neuroimage 14, 947-958. doi: 10.1006/nimg.2001.0892

den Ouden, D.-B., Saur, D., Mader, W., Schelter, B., Lukic, S., Wali, E., et al. (2012). Network modulation during complex syntactic processing. Neuroimage 59, 815-823. doi: 10.1016/j.neuroimage.2011.07.057

Depue, B. E., Orr, J. M., Smolker, H. R., Naaz, F., and Banich, M. T. (2016). The organization of right prefrontal networks reveals common mechanisms of inhibitory regulation across cognitive, emotional, and motor processes. Cereb. Cortex 26, 1634-1646. doi: 10.1093/cercor/bhu324

Deschamps, I., Baum, S. R., and Gracco, V. L. (2014). On the role of the supramarginal gyrus in phonological processing and verbal working memory: evidence from rTMS studies. Neuropsychologia 53, 39-46. doi: 10.1016/j.neuropsychologia.2013.10.015

D'Esposito, M. (2007). From cognitive to neural models of working memory. Philos. Trans. R. Soc. Lond. B Biol. Sci. 362, 761-772. doi: 10.1098/rstb.2007.2086

D’Esposito, M., Aguirre, G. K., Zarahn, E., Ballard, D., Shin, R. K., and Lease, J. (1998). Functional MRI studies of spatial and nonspatial working memory. Cogn. Brain Res. 7, 1-13. doi: 10.1016/S0926-6410(98)00004-4
Fehr, T., Code, C., and Herrmann, M. (2007). Common brain regions underlying different arithmetic operations as revealed by conjunct fMRI-BOLD activation. Brain Res. 1172, 93-102. doi: 10.1016/j.brainres.2007.07.043

Friederici, A. D. (2011). The brain basis of language processing: from structure to function. Physiol. Rev. 91, 1357-1392. doi: 10.1152/physrev.00006.2011

Fuertinger, S., Horwitz, B., and Simonyan, K. (2015). The functional connectome of speech control. PLoS Biol. 13:e1002209. doi: 10.1371/journal.pbio.1002209

Garcea, F. E., and Mahon, B. Z. (2014). Parcellation of left parietal tool representations by functional connectivity. Neuropsychologia 60, 131-143. doi: 10.1016/j.neuropsychologia.2014.05.018

Gierhan, S. M. E. (2013). Connections for auditory language in the human brain. Brain Lang. 127, 205-221. doi: 10.1016/j.bandl.2012.11.002

Gruber, O., and von Cramon, D. Y. (2003). The functional neuroanatomy of human working memory revisited: evidence from 3-T fMRI studies using classical domain-specific interference tasks. Neuroimage 19, 797-809. doi: 10.1016/S1053-8119(03)00089-2

Hanakawa, T., Dimyan, M. A., and Hallett, M. (2008). Motor planning, imagery, and execution in the distributed motor network: a time-course study with functional, MRI. Cereb. Cortex 18, 2775-2788. doi: 10.1093/cercor/bhn036

Hartwigsen, G., Baumgaertner, A., Price, C. J., Koehnke, M., Ulmer, S., and Siebner, H. R. (2010). Phonological decisions require both the left and right supramarginal gyri. Proc. Natl. Acad. Sci. U.S.A. 107, 16494-16499. doi: $10.1073 /$ pnas. 1008121107

Hasson, U., Skipper, J. I., Nusbaum, H. C., and Small, S. L. (2007). Abstract coding of audiovisual speech: beyond sensory representation. Neuron 56, 1116-1126. doi: 10.1016/j.neuron.2007.09.037

Hautzel, H., Mottaghy, F. M., Schmidt, D., Zemb, M., Shah, N. J., Müller-Gärtner, H. W., et al. (2002). Topographic segregation and convergence of verbal, object, shape and spatial working memory in humans. Neurosci. Lett. 323, 156-160. doi: 10.1016/S0304-3940(02)00125-8

Heim, S., Amunts, K., Hensel, T., Grande, M., Huber, W., Binkofski, F., et al. (2012). The role of human parietal area 7A as a link between sequencing in hand actions and in overt speech production. Front. Psychol. 3:534. doi: $10.3389 /$ fpsyg. 2012.00534

Henson, R. N. A., Shallice, T., and Dolan, R. J. (1999). Right prefrontal cortex and episodic memory retrieval: a functional MRI test of the monitoring hypothesis. Brain 122, 1367-1381. doi: 10.1093/brain/122.7.1367

Hertrich, I., Dietrich, S., and Ackermann, H. (2016). The role of the supplementary motor area for speech and language processing. Neurosci. Biobehav. Rev. 68, 602-610. doi: 10.1016/j.neubiorev.2016.06.030

Hétu, S., Grégoire, M., Saimpont, A., Coll, M.-P., Eugène, F., Michon, P.-E., et al. (2013). The neural network of motor imagery: an ALE meta-analysis. Neurosci. Biobehav. Rev. 37, 930-949. doi: 10.1016/j.neubiorev.2013.03.017

Higashiyama, Y., Takeda, K., Someya, Y., Kuroiwa, Y., and Tanaka, F. (2015). The neural basis of typewriting: a functional MRI study. PLoS ONE 10:e0134131. doi: 10.1371/journal.pone.0134131

Janata, P., and Grafton, S. T. (2003). Swinging in the brain: shared neural substrates for behaviors related to sequencing and music. Nat. Neurosci. 6, 682-687. doi: $10.1038 / \mathrm{nn} 1081$

Jefferies, E. (2013). The neural basis of semantic cognition: converging evidence from neuropsychology, neuroimaging and TMS. Cortex 49, 611-625. doi: 10.1016/j.cortex.2012.10.008

Johnson, M. R., and Johnson, M. K. (2014). Decoding individual natural scene representations during perception and imagery. Front. Hum. Neurosci. 8:59. doi: 10.3389/fnhum.2014.00059

Kelly, C., Uddin, L. Q., Shehzad, Z., Margulies, D. S., Castellanos, F. X., Milham, M. P., et al. (2010). Broca's region: linking human brain functional connectivity data and non-human primate tracing anatomy studies. Eur. J. Neurosci. 32, 383-398. doi: 10.1111/j.1460-9568.2010.07279.x

Koelsch, S. (2005). Neural substrates of processing syntax and semantics in music. Curr. Opin. Neurobiol. 15, 207-212. doi: 10.1016/j.conb.2005.03.005

Koelsch, S., Gunter, T. C., Cramon, D. Y., Zysset, S., Lohmann, G., and Friederici, A. D. (2002). Bach speaks: a cortical "language-network" serves the processing of music. Neuroimage 17, 956-966. doi: 10.1006/nimg.2002.1154

Kreifelts, B., Ethofer, T., Huberle, E., Grodd, W., and Wildgruber, D. (2010). Association of trait emotional intelligence and individual fMRI-activation patterns during the perception of social signals from voice and face. Hum. Brain Mapp. 31, 979-991. doi: 10.1002/hbm.20913 
Kunert, R., Willems, R. M., Casasanto, D., Patel, A. D., and Hagoort, P. (2015). Music and language syntax interact in broca's area: an fMRI study. PLoS ONE 10:e0141069. doi: 10.1371/journal.pone.0141069

Lahnakoski, J., Glerean, E., Salmi, J., Jääskeläinen, I., Sams, M., Hari, R., et al. (2012). Naturalistic fMRI mapping reveals superior temporal sulcus as the hub for the distributed brain network for social perception. Front. Hum. Neurosci. 6:233. doi: $10.3389 /$ fnhum.2012.00233

Langheim, F. J. P., Callicott, J. H., Mattay, V. S., Duyn, J. H., and Weinberger, D. R. (2002). Cortical systems associated with covert music rehearsal. Neuroimage 16, 901-908. doi: 10.1006/nimg.2002.1144

Langner, R., Sternkopf, M. A., Kellermann, T. S., Grefkes, C., Kurth, F., Schneider, F., et al. (2014). Translating working memory into action: behavioral and neural evidence for using motor representations in encoding visuospatial sequences. Hum. Brain Mapp. 35, 3465-3484. doi: 10.1002/hbm. 22415

Leaver, A. M., Van Lare, J., Zielinski, B., Halpern, A. R., and Rauschecker, J. P. (2009). Brain activation during anticipation of sound sequences. J. Neurosci. 29, 2477-2485. doi: 10.1523/JNEUROSCI.4921-08.2009

Leisman, G., Moustafa, A. A., and Shafir, T. (2016). Thinking, walking, talking: integratory motor and cognitive brain function. Front. Public Health 4:94. doi: 10.3389/fpubh.2016.00094

Li, Y., Long, J., Huang, B., Yu, T., Wu, W., Li, P., et al. (2016). Selective audiovisual semantic integration enabled by feature-selective attention. Sci. Rep. 6:18914. doi: $10.1038 /$ srep 18914

Martin, A. (2007). The representation of object concepts in the brain. Annu. Rev. Psychol. 58, 25-45. doi: 10.1146/annurev.psych.57.102904.190143

Meister, I. G., Krings, T., Foltys, H., Boroojerdi, B., Müller, M., Töpper, R., et al. (2004). Playing piano in the mind-an fMRI study on music imagery and performance in pianists. Cogn. Brain Res. 19, 219-228. doi: 10.1016/j.cogbrainres.2003.12.005

Musso, M., Weiller, C., Horn, A., Glauche, V., Umarova, R., Hennig, J., et al. (2015). A single dual-stream framework for syntactic computations in music and language. Neuroimage 117, 267-283. doi: 10.1016/j.neuroimage.2015. 05.020

Nachev, P., Kennard, C., and Husain, M. (2008). Functional role of the supplementary and pre-supplementary motor areas. Nat. Rev. Neurosci. 9, 856-869. doi: $10.1038 / \mathrm{nrn} 2478$

Nee, D. E., and D'Esposito, M. (2016). The hierarchical organization of the lateral prefrontal cortex. Elife 5:e12112. doi: 10.7554/eLife.12112

Noonan, K. A., Jefferies, E., Visser, M., and Lambon Ralph, M. A. (2013). Going beyond inferior prefrontal involvement in semantic control: evidence for the additional contribution of dorsal angular gyrus and posterior middle temporal cortex. J. Cogn. Neurosci. 25, 1824-1850. doi: 10.1162/jocn_a_00442

Nyberg, L., Habib, R., Tulving, E., Cabeza, R., Houle, S., Persson, J., et al. (2000). Large scale neurocognitive networks underlying episodic memory. J. Cogn. Neurosci. 12, 163-173. doi: 10.1162/089892900561805

Patel, A. D. (2003). Language, music, syntax and the brain. Nat. Neurosci. 6, 674-681. doi: $10.1038 / \mathrm{nn} 1082$

Planton, S., Jucla, M., Roux, F.-E., and Démonet, J.-F. (2013). The "handwriting brain": a meta-analysis of neuroimaging studies of motor versus orthographic processes. Cortex 49, 2772-2787. doi: 10.1016/j.cortex.2013.05.011

Radua, J., Del Pozo, N. O., Gómez, J., Guillen-Grima, F., and Ortuo, F. (2014). Meta-analysis of functional neuroimaging studies indicates that an increase of cognitive difficulty during executive tasks engages brain regions associated with time perception. Neuropsychologia 58, 14-22. doi: 10.1016/j.neuropsychologia.2014.03.016

Sato, K., Kirino, E., and Tanaka, S. (2015). A voxel-based morphometry study of the brain of university students majoring in music and non-music disciplines. Behav. Neurol. 2015, 9. doi: 10.1155/2015/ 274919

Schaal, N. K., Krause, V., Lange, K., Banissy, M. J., Williamson, V. J., and Pollok, B. (2015a). Pitch memory in nonmusicians and musicians: revealing functional differences using transcranial direct current stimulation. Cereb. Cortex 25, 2774-2782. doi: 10.1093/cercor/bhu0

Schaal, N. K., Williamson, V. J., Kelly, M., Muggleton, N. G., Pollok, B., Krause, V., et al. (2015b). A causal involvement of the left supramarginal gyrus during the retention of musical pitches. Cortex 64, 310-317. doi: 10.1016/j.cortex.2014.11.011
Schacter, D. L., Addis, D. R., Hassabis, D., Martin, V. C., Spreng, R. N., and Szpunar, K. K. (2012). The future of memory: remembering, imagining, and the brain. Neuron 76, 677-694. doi: 10.1016/j.neuron.2012.11.001

Schon, D., Anton, J. L., Roth, M., and Besson, M. (2002). An fMRI study of music sight-reading. Neuroreport 13, 2285-2289. doi: 10.1097/00001756-200212030-00023

Simmonds, A. J., Wise, R. J., Collins, C., Redjep, O., Sharp, D. J., Iverson, P., et al. (2014). Parallel systems in the control of speech. Hum. Brain Mapp. 35, 1930-1943. doi: 10.1002/hbm.22303

Simonyan, K., and Fuertinger, S. (2015). Speech networks at rest and in action: interactions between functional brain networks controlling speech production. J. Neurophysiol. 113, 2967-2978. doi: 10.1152/jn.00964.2014

Solodkin, A., Hlustik, P., Chen, E. E., and Small, S. L. (2004). Fine modulation in network activation during motor execution and motor imagery. Cereb. Cortex 14, 1246-1255. doi: 10.1093/cercor/bhh086

Spielberg, J. M., Miller, G. A., Heller, W., and Banich, M. T. (2015). Flexible brain network reconfiguration supporting inhibitory control. Proc. Natl. Acad. Sci. U.S.A. 112, 10020-10025. doi: 10.1073/pnas.1500048112

Spiridon, M., Fischl, B., and Kanwisher, N. (2006). Location and spatial profile of category-specific regions in human extrastriate cortex. Hum. Brain Mapp. 27, 77-89. doi: 10.1002/hbm.20169

Spreng, R. N., Sepulcre, J., Turner, G. R., Stevens, W. D., and Schacter, D. L. (2013). Intrinsic architecture underlying the relations among the default, dorsal attention, and frontoparietal control networks of the human brain. J. Cogn. Neurosci. 25, 74-86. doi: 10.1162/jocn_a_00281

Suchan, B., Botko, R., Gizewski, E., Forsting, M., and Daum, I. (2006). Neural substrates of manipulation in visuospatial working memory. Neuroscience 139, 351-357. doi: 10.1016/j.neuroscience.2005.08.020

Takahashi, H., Shibuya, T., Kato, M., Sassa, T., Koeda, M., Yahata, N., et al. (2008). Enhanced activation in the extrastriate body area by goal-directed actions. Psychiatry Clin. Neurosci. 62, 214-219. doi: 10.1111/j.1440-1819.2008.01757.x

Tanaka, S., and Kirino, E. (2016). Functional connectivity of the precuneus in female university students with long-term musical training. Front. Hum. Neurosci. 10:328. doi: 10.3389/fnhum.2016.00328

Tanaka, S., and Kirino, E. (2017). Reorganization of the thalamocortical network in musicians. Brain Res. 1664, 48-54. doi: 10.1016/j.brainres.2017.03.027

Tomasino, B., and Gremese, M. (2016). The cognitive side of M1. Front. Hum. Neurosci. 10:298. doi: 10.3389/fnhum.2016.00298

Tune, S., and Asaridou, S. S. (2016). Stimulating the semantic network: what can TMS tell us about the roles of the posterior middle temporal gyrus and angular gyrus? J. Neurosci. 36, 4405-4407. doi: 10.1523/JNEUROSCI.0194-16.2016

Turkeltaub, P. E., and Coslett, H. B. (2010). Localization of sublexical speech perception components. Brain Lang. 114, 1-15. doi: 10.1016/j.bandl.2010.03.008

van Elk, M., van Schie, H., and Bekkering, H. (2014). Action semantics: a unifying conceptual framework for the selective use of multimodal and modality-specific object knowledge. Phys. Life Rev. 11, 220-250. doi: 10.1016/j.plrev.2013.11.005

Vesia, M., and Crawford, J. D. (2012). Specialization of reach function in human posterior parietal cortex. Exp. Brain Res. 221, 1-18. doi: $10.1007 / \mathrm{s} 00221-012-3158-9$

Whitfield-Gabrieli, S., and Nieto-Castanon, A. (2012). Conn: a functional connectivity toolbox for correlated and anticorrelated brain networks. Brain Connect. 2, 125-141. doi: 10.1089/brain.2012.0073

Whitney, C., Kirk, M., O’Sullivan, J., Lambon Ralph, M. A., and Jefferies, E. (2011a). Executive semantic processing is underpinned by a large-scale neural network: revealing the contribution of left prefrontal, posterior temporal, and parietal cortex to controlled retrieval and selection using, TMS. J. Cogn. Neurosci. 24, 133-147. doi: 10.1162/jocn_a_00123

Whitney, C., Kirk, M., O'Sullivan, J., Lambon Ralph, M. A., and Jefferies, E. (2011b). The neural organization of semantic control: TMS evidence for a distributed network in left inferior frontal and posterior middle temporal gyrus. Cereb. Cortex 21, 1066-1075. doi: 10.1093/cercor/bhq18

Xiang, H.-D., Fonteijn, H. M., Norris, D. G., and Hagoort, P. (2010). Topographical functional connectivity pattern in the perisylvian language networks. Cereb. Cortex 20, 549-560. doi: 10.1093/cercor/bhp119

Zarate, J. M., and Zatorre, R. J. (2008). Experience-dependent neural substrates involved in vocal pitch regulation during singing. Neuroimage 40, 1871-1887. doi: 10.1016/j.neuroimage.2008.01.026 
Zatorre, R. J., Halpern, A. R., and Bouffard, M. (2010). Mental reversal of imagined melodies: a role for the posterior parietal cortex. J. Cogn. Neurosci. 22, 775-789. doi: 10.1162/jocn.2009.21239

Zevin, J. D., Yang, J., Skipper, J. I., and McCandliss, B. D. (2010). Domain general change detection accounts for "dishabituation" effects in temporal-parietal regions in functional magnetic resonance imaging studies of speech perception. J. Neurosci. 30, 1110-1117. doi: 10.1523/JNEUROSCI.4599-09.2010

Zhang, S., Ide, J. S., and Li, C.-S. R. (2012). Resting-state functional connectivity of the medial superior frontal cortex. Cereb. Cortex 22, 99-111. doi: $10.1093 /$ cercor/bhr088
Conflict of Interest Statement: The authors declare that the research was conducted in the absence of any commercial or financial relationships that could be construed as a potential conflict of interest.

Copyright (๑) 2017 Tanaka and Kirino. This is an open-access article distributed under the terms of the Creative Commons Attribution License (CC BY). The use, distribution or reproduction in other forums is permitted, provided the original author(s) or licensor are credited and that the original publication in this journal is cited, in accordance with accepted academic practice. No use, distribution or reproduction is permitted which does not comply with these terms. 